\title{
An Inequality for Algebroid Functions and Some Applications
}

\section{Ángel J. Alonso Gómez}

\begin{abstract}
In this paper we present a version for algebroid functions of an inequality due to Barsegian (Math URSS Sbornik 42(2):155, 1982) and we get two consequences: first a kind of deficiency relation for curves (preimages of an algebroid function, the so-called $\Gamma$-lines) which is an extension of the well-known relation of Barsegian ( $\Gamma$ lines. On the geometry of real and complex functions. Taylor \& Francis, London, 2002) and secondly a proximity principle for the preimages of an algebroid function, also an extension of the principle for meromorphic functions.
\end{abstract}

Keywords Algebroid functions · Gamma-lines · Deficiency relations · Proximity principles

Mathematics Subject Classification 30C99

\section{Algebroid Functions}

An algebroid function is a multivaluated one $w=w(z)$ with a finite number of branches in $\mathbb{C}$ and whose only singularities are poles and algebraic branch points $[10,11]$. These functions satisfy an equation like

$$
w^{k}+A_{1}(z) w^{k-1}+\cdots+A_{k-1}(z) w+A_{k}(z)=0
$$


with $A_{i}, i=1,2, \ldots, k$ meromorphic functions.

$k$ is the order of the algebroid function.

But we can also consider algebroid functions as holomorphic functions defined on a Riemann surface with $k$ sheets [4]: we have the Riemann surface

$$
\Xi=\left\{\left(z, w_{i}(z)\right), z \in \mathbb{C}, i=1,2, \ldots, k\right\}
$$

where $w_{1}, w_{2}, \ldots w_{k}$ are the branches of the algebroid function.

The canonical projection

$$
\begin{gathered}
p: \Xi \rightarrow \mathbb{C} \\
\left(z, w_{i}(z)\right) \rightarrow z
\end{gathered}
$$

is an holomorphic covering of $\mathbb{C}$ and

$$
\begin{aligned}
f: \Xi & \rightarrow \widehat{\mathbb{C}} \\
\left(z, w_{i}(z)\right) & \rightarrow w_{i}(z)
\end{aligned}
$$

is also holomorphic. It is the function associated to the algebroid function.

\section{The Inequality}

We can consider the relation

$$
\begin{aligned}
& \underset{\Xi \rightarrow \widehat{C}}{f} \\
& p \underset{\mathbb{C}}{\downarrow} w_{i}
\end{aligned}
$$

between the function associated to the algebroid function and its branches. $\Xi$ is now a Riemann surface of $k$ sheets and $p$ is locally the identity.

Then

$$
w_{i} \circ p=f
$$

but $p^{\prime}=1$ and $p^{\prime \prime}=0 \Longrightarrow f^{\prime \prime}=w_{i}^{\prime \prime} \circ p$.

From now on $\Xi[r]$ is the part of the Riemann surface $\Xi$ which projects by means of $p$ onto $D[r]$, the closed disk of $\mathbb{C}$ centered in $(0,0)$, with radius $r$.

$$
\begin{gathered}
p: \Xi \rightarrow \mathbb{C} \\
p(\zeta)=z
\end{gathered}
$$

and

$$
\Phi=p^{*}(r d r d \varphi)
$$


is the pull-back of the element of surface on the plane to the surface $\Xi$.

\section{Proposition 1}

$$
\underset{\Xi[r]}{\iint^{\prime \prime}(\zeta)}\left|\frac{f^{\prime \prime}(\zeta)}{f^{\prime}(\zeta)}\right| \Phi \sum_{i=1}^{k} \iint_{D[r]}\left|\frac{w_{i}^{\prime \prime}(z)}{w_{i}^{\prime}(z)}\right| r d r d \varphi
$$

with $z=r e^{i \varphi}$.

Proof Around any point of $D[r]$ there is a neighbourhood $U_{i}$ with $p^{-1}\left(U_{i} \cap D[r]\right) \subset$ $\Xi[r]$ and a $w_{i}$ defined in $U_{i} \cap D[r]$.

$p$ is locally an homeomorphism (it is the identity indeed) and applies every neighbourhood in $\Xi[r]$ onto a neighbourhood in $D[r]$.

Then, if around every point of $D[r], z=p(\zeta)$ there is a neighbourhood $U_{i}$ where a branch $w_{i}$ of $f$ is defined, $w_{1}, w_{2}, \ldots, w_{k}$ are defined in $U_{1} \cap D[r], U_{2} \cap D[r]$, $\ldots$, in $U_{k} \cap D[r]$, in such a way that

$$
\bigcup_{i=1}^{k}\left(U_{i} \cap D[r]\right)=D[r]
$$

then

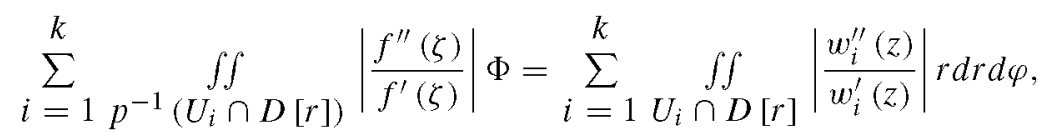

but

$$
\underset{\Xi[r]}{\left.\iint^{\prime \prime} \mid \zeta\right)}\left|\frac{f^{\prime \prime}(\zeta)}{f^{\prime}(\zeta)}\right| \sum_{i=1}^{k} \underset{p^{-1}\left(U_{i} \cap D[r]\right)}{\int f_{f^{\prime}(\zeta)}} \mid \frac{f^{\prime \prime}(\zeta)}{\mid \Phi}
$$

hence

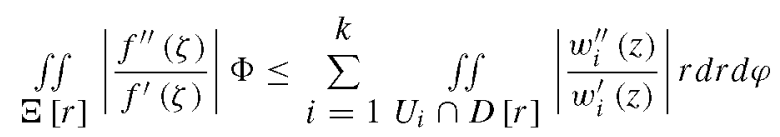

But this argument only is valid with only one sheet in the surface $\Xi, p$ being a homeomorphism only locally because it projects points $\zeta$ in the different sheets of $\Xi$ onto the same point $z \in \mathbb{C}$, hence we must consider the totality of the sheets and we get

$$
\iint_{\Xi[r]}\left|\frac{f^{\prime \prime}(\zeta)}{f^{\prime}(\zeta)}\right| \Phi \leq k \sum_{i=1}^{k} \underset{U_{i} \cap D[r]}{\iint_{i}}\left|\frac{w_{i}^{\prime \prime}(z)}{w_{i}^{\prime}(z)}\right| r d r d \varphi
$$


finally, we observe that

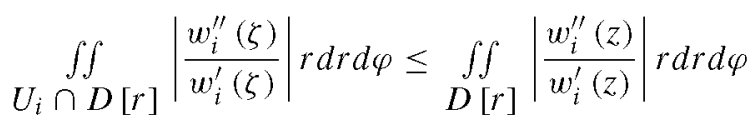

and we finally get

$$
\underset{\Xi[r]}{\iint}\left|\frac{f^{\prime \prime}(\zeta)}{f^{\prime}(\zeta)}\right| \Phi \leq k \sum_{i=1}^{k} \iint_{D[r]}\left|\frac{w_{i}^{\prime \prime}(z)}{w_{i}^{\prime}(z)}\right| r d r d \varphi
$$

Observation: arguing in the same way for the integral

$$
A(r)=\frac{1}{\pi} \underset{\Xi[r]}{ } \frac{\left|f^{\prime}(\zeta)\right|^{2}}{\left(1+\left|f^{\prime}(\zeta)\right|^{2}\right)^{2}} \Phi
$$

which is the density of the well-known Ahlfors-Shimizu characteristic, we get

$$
A(r) \leq k \sum_{i=1}^{k} \iint_{D[r]} \frac{\left|w_{i}^{\prime}(z)\right|^{2}}{\left(1+\left|w_{i}^{\prime}(z)\right|^{2}\right)^{2}} r d r d \varphi
$$

and we shall call, from now on

$$
A_{i}(r)=\iint_{D[r]} \frac{\left|w_{i}^{\prime}(z)\right|^{2}}{\left(1+\left|w_{i}^{\prime}(z)\right|^{2}\right)^{2}} r d r d \varphi
$$

$A(r)$ is the Ahlfors-Shimizu density of the Riemannian image of $\Xi[r]$ under $f$ and $A_{i}(r)$ those of $D[r]$ under the branches of the algebroid function.

\section{The Defect Relation}

Let $\Gamma_{v}, v=1,2, \ldots, q$ be $q$ Jordan, smooth, disjoint, bounded curves, with $v\left(\Gamma_{\nu}\right)<$ $\infty$, where $v(\Gamma)=\operatorname{Var}_{\zeta \in \Gamma} \alpha_{\Gamma}(\zeta)$, being $\alpha_{\Gamma}(\zeta)$ the angle between the tangent to $p(\Gamma)$ in $p(\zeta)$ and the $O X$ axis. We add the hypothesis that $f^{-1}\left(\Gamma_{\nu}\right)$ does not contain poles nor branch points of $\Xi$.

Def.- $\Delta(\Gamma)=\liminf _{r \rightarrow \infty} \frac{\Lambda(r, \Gamma)}{r \sum_{i=1}^{k} A_{i}(r)}$, where $\Lambda(r, \Gamma)$ is the length of the preimages of $\Gamma$ in $\Xi[r]$, under the algebroid function $f$.

$\Delta(\Gamma)$ is the deficiency of $\Gamma$ and we shall call $\Gamma$ a deficient curve if and only if $\Delta(\Gamma) \neq 0$.

With this notations, we have the 
Theorem 1 Let $\Gamma_{v}, v=1,2, \ldots, q$ be a collection of curves as above and let $\Delta\left(\Gamma_{v}\right)$ be the deficiency of every $\Gamma_{v}$, then:

$$
\sum_{v=1}^{q} \Delta\left(\Gamma_{\nu}\right) \leq C k
$$

where $C$ is an absolute constant and $k$ is the order of the algebroid function.

Proof We begin with

$$
\sum_{\nu=1}^{q} \Lambda\left(r, \Gamma_{\nu}\right) \leq K \iint_{\Xi[r]}\left|\frac{f^{\prime \prime}(\zeta)}{f^{\prime}(\zeta)}\right| \Phi+h \pi r \sqrt{k A(r)}+2 \sqrt{2} k \pi r
$$

$K$ and $h$ being independent of the function $f$. [1]

Then

$$
\sum_{\nu=1}^{q} \Lambda\left(r, \Gamma_{\nu}\right) \leq K \underset{D[r]}{k \int}\left|\frac{w_{i}^{\prime \prime}(z)}{w_{i}^{\prime}(z)}\right| r d r d \varphi+h \pi r \sqrt{k A(r)}+2 \sqrt{2} k \pi r
$$

but

$$
\iint_{D[r]}\left|\frac{w_{i}^{\prime \prime}(z)}{w_{i}^{\prime}(z)}\right| r d r d \varphi \leq \widehat{K} r A_{i}(r)
$$

for $r \in E_{i} \subset \mathbb{R}$ such that $\liminf _{r \rightarrow \infty} \frac{1}{r} \int_{E_{i} \cap[0, \infty)} d \ln t=C_{i} \in(0,1)$, i. e. $E_{i}$ being a set of lower logarithmic density $C_{i}$. (Branches $w_{i}$ are meromorphic functions) [3].

Then

$$
\sum_{v=1}^{q} \Lambda\left(r, \Gamma_{v}\right) \leq K k r \sum_{i=1}^{k} A_{i}(r)+h \pi r \sqrt{k A(r)}+2 \sqrt{2} k \pi r
$$

but

$$
\sqrt{k A(r)} \leq k A(r) \leq k^{2} \sum_{i=1}^{k} A_{i}(r)
$$

and we get

$$
\sum_{\nu=1}^{q} \Lambda\left(r, \Gamma_{\nu}\right) \leq K k r \sum_{i=1}^{k} A_{i}(r)+h \pi r k^{2} \sum_{i=1}^{k} A_{i}(r)+2 \sqrt{2} k \pi r
$$


and finally

$$
\sum_{\nu=1}^{q} \Lambda\left(r, \Gamma_{\nu}\right) \leq k r\left[C_{1} \sum_{i=1}^{k} A_{i}(r)+C_{2}\right]
$$

with $r \in E$ as above.

Then, with the definition of $\Delta(\Gamma)$ we get the proposition we were looking for.

Now, if we have $q \rightarrow \infty$ we get

$$
\sum_{\nu=1}^{\infty} \Delta\left(\Gamma_{\nu}\right) \leq C k
$$

and it remains bounded, so that we must have $\Delta(\Gamma)=0$ for almost all curves and only a numerable quantity of curves are such that $\Delta(\Gamma)>0$. Those are the deficient curves.

\section{The Proximity Principle}

We are now speaking about the problem of the distribution of preimages of a function (an algebroid one, in our case) and about an estimation of their proximity. If we call $a$-points corresponding to $z$ those that satisfies $f(z)=a$, we will try to progress in the direction of the so-called Littlewood Property [6] which this author has asserted for entire functions and says, roughly speaking, that almost all $a$-points of those functions, for almost all values of $a$, belong to a subset of $\mathbb{C}$ of small area. Littlewood has proved that the property is true under the hypothesis that the bound

$$
\iint_{D(1)} \frac{\left|p^{\prime}(z)\right|}{1+\left|p(z)^{2}\right|} r d r d \varphi \leq c n^{\frac{1}{2}-\alpha}
$$

is fulfilled by a polinomial $p$ of degree less or equal than $n$ and $\alpha>0$.

This is the conjecture of Littlewood, proved by Lewis and $\mathrm{Wu}$ [7].

In this direction, we extend a result of Barsegian [Bar1] for meromorphic functions to algebroid functions.

Let $a_{v}, b_{v}, v=1,2 \ldots, q$ be values in $\mathbb{C}, f$ an algebroid function, $\zeta_{i}(z)$ are the preimages of $z$, i. e. $f\left(\zeta_{i}(z)\right)=z . n(r, z)$ is the number of preimages of $z$ in $\Xi[r]$.

Let $\Gamma_{\nu}$ be Jordan, bounded, smooth, disjoint, bounded curves, with $v\left(\Gamma_{\nu}\right)<\infty$, and the extreme points of $\Gamma_{v}$ are $a_{v}$ and $b_{v}$.

$f^{-1}\left(\Gamma_{\nu}\right) \cap \Xi[r]$ contains $n\left(r, a_{\nu}\right)$ curves $\gamma_{i}, i=1,2, \ldots, n\left(r, a_{\nu}\right)$ with only ordinary points, i. e. they do not pass through singular points.

We assign to the preimages of the extreme points $a_{v}$ and $b_{v}$ the same index, $\zeta_{i}\left(a_{v}\right), \zeta_{i}\left(b_{v}\right)$ respectively. The other points from $n\left(r, a_{v}\right)$ to $n\left(r, b_{v}\right)$ remains arbitrary. 
So, we define

$$
B(r, a, b)=\sum_{i=1}^{n(r, a)} \operatorname{dist}\left(\zeta_{i}(a), \zeta_{i}(b)\right)
$$

where

$$
\operatorname{dist}\left(\zeta_{i}(a), \zeta_{i}(b)\right)=\inf \underset{\gamma\left(\zeta_{i}(a), \zeta_{i}(b)\right)}{\int} d s
$$

being $d s$ the metric on the surface $\Xi$ and $\gamma(\alpha, \beta)$ those regular curves joining the points $\alpha$ and $\beta$ and

$$
A(r, a, b)=\sum_{i=n(r, a)+1}^{n(r, b)}\left|z_{i}(b)\right|
$$

with $z_{i}(b)=p\left(\zeta_{i}(b)\right)$.

The proximity principle is given by the next

Theorem 2 There exist $K$, and $C \in(0,1)$ absolute constants such that for every $r \in E \subset[0, \infty)$ of lower logarithmic density $C$

$$
\sum_{\nu=1}^{q}\left|A\left(r, a_{\nu} \cdot b_{\nu}\right)+B\left(r, a_{\nu}, b_{\nu}\right)\right| \leq K r\left[A(r)+\sum_{i=1}^{k} A_{i}(r)\right]+O(r A(r))
$$

where $A(r)$ and $A_{i}(r)$ are as above.

Proof The extreme points of $\gamma_{i}$ are $a$-points and $b$-points, $\zeta_{i}(a)$ and $\zeta_{i}(b)$ respectively, then there are $n\left(r, a_{\nu}\right)$ pairs $\left(\zeta_{i}\left(a_{\nu}\right), \zeta_{i}\left(b_{\nu}\right)\right), i=1,2, \ldots, n\left(r, a_{\nu}\right)$ and the remain are summed in an arbitrary manner.

Let $F_{r}$ be the riemmanian image of $\Xi[r]$ under $f$. There are $n_{0}(r, \Gamma)$ simple islands of $F_{r}$ over $\Gamma[5]$ and $\tilde{n}(r, \Gamma)$ multiple islands (any of them counted only once).

Now, if $n(r, \Gamma)$ is the sum of the multiplicities of all islands over $\Gamma$, we have

$$
n(r, \Gamma)=n_{0}(r, \Gamma)+n_{1}(r, \Gamma)+\tilde{n}(r, \Gamma)
$$

where $n_{1}(r, \Gamma)$ is the sum of the orders of all islands over $\Gamma$.

Then

$$
A(r)-n(r, \Gamma)=A(r)-n_{0}(r, \Gamma)-n_{1}(r, \Gamma)-\tilde{n}(r, \Gamma)
$$


and so

$$
A(r)-n(r, \Gamma)+n_{1}(r)=A(r)-n_{0}(r, \Gamma)-\tilde{n}(r, \Gamma)
$$

but, by the second fundamental theorem of Ahlfors [9]

$$
\sum_{\nu=1}^{q} n\left(r, \Gamma_{\nu}\right)-\sum_{\nu=1}^{q} n_{1}\left(r, \Gamma_{v}\right) \geq(q-2) A(r)-h L(r)
$$

where $h$ is a constant depending on the curves and $L(r)$ is the length of the relative boundary of $F_{r}$ and consequently

$$
h L(r)=o(A(r))
$$

then

$$
\sum_{\nu=1}^{q}\left(A(r)-n_{0}\left(r, \Gamma_{\nu}\right)-\tilde{n}\left(r, \Gamma_{\nu}\right)\right) \leq 2 A(r)+h L(r)
$$

but, from the first fundamental theorem of Ahlfors [9] we can deduce

$$
\sum_{\nu=1}^{q} n\left(r, \Gamma_{\nu}\right) \leq q A(r)+h L(r)
$$

then

$$
\sum_{\nu=1}^{q} n_{0}\left(r, \Gamma_{\nu}\right)+\sum_{\nu=1}^{q} n_{1}\left(r, \Gamma_{\nu}\right)+\sum_{\nu=1}^{q} \tilde{n}\left(r, \Gamma_{\nu}\right) \leq q A(r)+h L(r)
$$

and consequently

$$
\sum_{\nu=1}^{q} \tilde{n}\left(r, \Gamma_{\nu}\right) \leq q A(r)-\sum_{\nu=1}^{q} n_{0}\left(r, \Gamma_{\nu}\right)-\sum_{\nu=1}^{q} n_{1}\left(r, \Gamma_{\nu}\right)+h L(r)
$$

but $\tilde{n}<n_{1}$, hence

$$
\sum_{\nu=1}^{q} \tilde{n}\left(r, \Gamma_{\nu}\right) \leq q A(r)-\sum_{\nu=1}^{q} n_{0}\left(r, \Gamma_{\nu}\right)-\sum_{\nu=1}^{q} \tilde{n}\left(r, \Gamma_{\nu}\right)+h L(r)
$$


and

$$
q A(r)-\sum_{\nu=1}^{q} \tilde{n}\left(r, \Gamma_{\nu}\right)-\sum_{\nu=1}^{q} n_{0}\left(r, \Gamma_{\nu}\right) \leq 2 A(r)+h L(r)
$$

so that

$$
\sum_{\nu=1}^{q} \tilde{n}\left(r, \Gamma_{\nu}\right) \leq 2 A(r)+h L(r)
$$

with different constant $h$ and, by

$$
\sum_{\nu=1}^{q}\left(A(r)-n\left(r, \Gamma_{\nu}\right)\right)=\sum_{\nu=1}^{q}\left(A(r)-n_{0}\left(r, \Gamma_{\nu}\right)-n_{1}\left(r, \Gamma_{\nu}\right)-\tilde{n}\left(r, \Gamma_{\nu}\right)\right)
$$

we get

$$
\sum_{\nu=1}^{q}\left(A(r)-n_{0}\left(r, \Gamma_{\nu}\right)\right) \leq 4 A(r)+h L(r)
$$

with different $h$ again and

$$
\sum_{\nu=1}^{q}\left(n_{1}\left(r, \Gamma_{\nu}\right)+\tilde{n}\left(r, \Gamma_{\nu}\right)\right) \leq 4 A(r)-\sum_{\nu=1}^{q}\left(A(r)-n\left(r, \Gamma_{\nu}\right)\right)+h L(r)
$$

but Miles [8] has proved that

$$
\sum_{\nu=1}^{q}\left|A(r)-n\left(r, a_{\nu}\right)\right| \leq K A(r), \quad r \rightarrow \infty
$$

with $r \in E$, a subset of $[0, \infty)$ with lower logarithmic density $C \in(0,1)$ and every $a_{\nu}$ belongs to a $\Gamma_{v}$ so that

$$
\sum_{\nu=1}^{q}\left|A(r)-n\left(r, \Gamma_{\nu}\right)\right| \leq K A(r), \quad r \rightarrow \infty \quad r \in E
$$

and the same is true for $n\left(r, b_{\nu}\right)$, hence

$$
\sum_{\nu=1}^{q}\left(n_{1}\left(r, a_{\nu}\right)+\tilde{n}\left(r, a_{\nu}\right)\right) \leq 4 A(r)-\sum_{\nu=1}^{q}\left(A(r)-n\left(r, a_{\nu}\right)\right)+h L(r)
$$




$$
\sum_{\nu=1}^{q}\left(n_{1}\left(r, b_{\nu}\right)+\tilde{n}\left(r, b_{\nu}\right)\right) \leq 4 A(r)-\sum_{\nu=1}^{q}\left(A(r)-n\left(r, b_{\nu}\right)\right)+h L(r)
$$

and we get

$$
\sum_{\nu=1}^{q}\left(n_{1}\left(r, a_{\nu}\right)+n_{1}\left(r, b_{\nu}\right)+\tilde{n}\left(r, a_{\nu}\right)+\tilde{n}\left(r, b_{\nu}\right)\right) \leq 8 A(r)+2 K A(r)+h L(r)
$$

always with $r \rightarrow \infty, \quad r \in E$.

Over $\Gamma_{\nu}$ there are $n\left(r, \Gamma_{\nu}\right)$ simple islands of $F_{r}$. Their preimages are $\gamma_{i}(\nu)$, $i=1,2, \ldots, n_{0}\left(r, \Gamma_{\nu}\right)$ and $n_{0}\left(r, \Gamma_{\nu}\right) \leq n\left(r, a_{\nu}\right)$.

The extreme points of $\gamma_{i}(v) \subset \Xi[r]$ are not multiple points, but ordinary points, namely $\zeta_{i}\left(a_{v}\right)$ and $\zeta_{i}\left(b_{v}\right)$ and there are $n_{0}\left(r, \Gamma_{v}\right)$ pairs of points of this kind and

$$
\sum_{i=1}^{n_{0}\left(r, \Gamma_{\nu}\right)} \operatorname{length}\left(\gamma_{i}(v)\right) \leq \Lambda\left(r, \Gamma_{v}\right)
$$

where $\Lambda\left(r, \Gamma_{v}\right)$ is the sum of the lengths of preimages of $f$.

And so

$$
\sum_{i=1}^{n_{0}\left(r, \Gamma_{\nu}\right)} \operatorname{dist}\left(\zeta_{i}\left(a_{\nu}\right), \zeta_{i}\left(b_{\nu}\right)\right) \leq \Lambda\left(r, \Gamma_{\nu}\right)
$$

It is obvious that

$$
\begin{aligned}
& \sum_{i=}^{n\left(r, a_{\nu}\right)} n_{0}\left(r, \Gamma_{\nu}\right)+1 \\
& \leq r\left(n\left(r, a_{\nu}\right)-n_{0}\left(r, \Gamma_{\nu}\right)+n\left(r, b_{\nu}\right)-n_{0}\left(r, \Gamma_{\nu}\right), \zeta_{i}\left(b_{\nu}\right)\right)+A\left(r, a_{\nu}, b_{\nu}\right) \leq
\end{aligned}
$$

hence

$$
\sum_{i=n_{o}\left(r, \Gamma_{\nu}\right)+1}^{n\left(r, a_{\nu}\right)} \operatorname{dist}\left(\zeta_{i}\left(a_{\nu}\right), \zeta_{i}\left(b_{\nu}\right)\right)+A\left(r, a_{\nu}, b_{\nu}\right) \leq 2 r\left(n\left(r, \Gamma_{\nu}\right)-n_{0}\left(r, \Gamma_{\nu}\right)\right)
$$

and

$$
A\left(r, a_{\nu, b_{\nu}}\right)+B\left(r, a_{\nu}, b_{\nu}\right)=\sum_{i=1}^{n_{0}\left(r, \Gamma_{\nu}\right)} \operatorname{dist}\left(\zeta_{i}\left(a_{\nu}\right), \zeta_{i}\left(b_{\nu}\right)\right)
$$




$$
\begin{gathered}
\quad \sum_{n_{0}\left(r, \Gamma_{\nu}\right)+1}^{n\left(r, \Gamma_{\nu}\right)} \operatorname{dist}\left(\zeta_{i}\left(a_{\nu}\right), \zeta_{i}\left(b_{\nu}\right)\right)+A\left(r, a_{\nu}, b_{\nu}\right) \\
\Rightarrow \sum_{\nu=1}^{q}\left|A\left(r, a_{\nu}, b_{\nu}\right)+B\left(r, a_{\nu}, b_{\nu}\right)\right| \leq \sum_{\nu=1}^{q} \Lambda\left(r, \Gamma_{\nu}\right)+(8+2 K) r A(r) h r L(r)
\end{gathered}
$$

but we saw previously that

$$
\sum_{\nu=1}^{q} \Lambda\left(r, \Gamma_{\nu}\right) \leq k r\left[C_{1} \sum_{i=1}^{k} A_{i}(r)+C_{2}\right]
$$

and finally

$$
\begin{aligned}
& \sum_{\nu=1}^{q}\left|\left(r, a_{\nu}, b_{v}\right)+B\left(r, a_{\nu}, b_{\nu}\right)\right| \\
& \leq k r\left(C_{1} \sum_{i=1}^{k} A_{i}(r)+C_{2}\right)+(8+2 K) r A(r)+h r L(r)
\end{aligned}
$$

\section{References}

1. Alonso Gómez, A., Fernández Arias, A.: $\Gamma$-lines of algebroid functions. Complex Anal. Oper. Theory 5, 847-861 (2011)

2. Barsegian, G.: $\Gamma$-Lines. On the Geometry of Real and Complex Functions. Taylor \& Francis, London (2002)

3. Barsegian, G.: On the geometry of meromorphic functions. Math. URSS Sbornik 42(2), 155 (1982)

4. Fernández Arias, A.: The error term in nevanlinna second fundamental theorem for holomorphic mappings on coverings. Rev. R. Acad. Ciencias. Ser. Mat. 96(1), 7-21 (2002)

5. Hayman, W.: Meromorphic Functions. Clarendon Press, Oxford (1975)

6. Littlewood, J.E.: On some conjectured inequalities with applications to the theory of integral functions. J. Lond. Math. Soc. 27, 387-393 (1952)

7. Lewis, J.L., Wu, Y.-M.: On conjectures of Arakelyan and Littlewood. J. Anal. Math. 50, 259-283 (1988)

8. Miles, J.: Bounds of the ratio $\frac{n(r, a)}{A(r)}$ for meromorphic functions. Trans. Am. Math. Soc. 162, 383-393 (1971)

9. Rolf, N.: Analytic Functions. Springer, New York (1970)

10. Remoundos, G.: Extension aux fonctions algébroïdes multiformes du théorème de M. Picard et de ses généralisations. Mémorial des sciences mathématiques. Gauthier-Villars, Paris (1927)

11. Selberg, H.L.: Algebroide Funktionen und umkehrfunktionen Abelscher Integrale. Avk. Norske Vid.Acad. Oslo Mat-naturvid. Kl(8), 1-72 (1934) 